\title{
Diuretic Renal Scintigraphy in Patients with Sulfonamide Allergies: Possible Alternative Use of Ethacrynic Acid
}

\author{
Ba D. Nguyen ${ }^{1}$, Michael C. Roarke ${ }^{1}$, Jason Robert Young ${ }^{2}$, Ming Yang ${ }^{1}$, and Howard H. Osborn ${ }^{1}$ \\ ${ }^{I}$ Department of Radiology, Mayo Clinic, Scottsdale, Arizona; and ${ }^{2}$ Department of Radiology, Maricopa Integrated Health System, \\ Phoenix, Arizona
}

Furosemide may trigger life-threatening sulfonamide crosshypersensitivity reactions, posing a dilemma in patients who need diuretic renal scintigraphy. Methods: We present our experience using ethacrynic acid as a nonsulfonamide alternative diuretic in 5 patients, with a discussion of the diuretic molecular structure, potential side effects, protocol development, and imaging results. Results: Diuretic renal scintigraphy using ethacrynic acid provided useful information about the obstruction status in all patients, with no adverse clinical impact. Conclusion: Ethacrynic acid is a potential alternative to furosemide for patients with severe sulfonamide reactions.

Key Words: ethacrynic acid; diuretic renal scintigraphy; sulfonamide allergy

J Nucl Med Technol 2015; 43:239-241

DOI: $10.2967 /$ jnmt.115.161331

$\mathbf{T}$

he objective of this essay is to raise awareness of ethacrynic acid as a possible alternative to furosemide for provocative diuretic renal scintigraphy. We retrospectively report on our experience with 5 patients, describing protocol development, patient safety, and diagnostic value with regard to conventional diuretic renal scintigraphy.

\section{MATERIALS AND METHODS}

Our institutional review board approved this retrospective study, and the requirement to obtain informed consent was waived. Between October 2009 and May 2014, 3 male and 2 female patients underwent diuretic renal scintigraphy using ethacrynic acid as an alternative to furosemide. Each patient had known prior severe sulfonamide reactions, including hives, tongue swelling, respiratory distress, vomiting, oral pruritus, and giant hives. The patient's ages were $25,54,66,83$, and $84 \mathrm{y}$. Three patients presented with hydronephrosis and recurrent urinary tract infections, the fourth with remote pyeloplasty and intermittent flank pain, and

Received May 24, 2015; revision accepted Sep. 2, 2015.

For correspondence or reprints contact: Ba D. Nguyen, Department of Radiology, Mayo Clinic, 13400 E. Shea Blvd., Scottsdale, AZ 85259.

E-mail: nguyen.ba@mayo.edu

Published online Oct. 15, 2015.

COPYRIGHT (c) 2015 by the Society of Nuclear Medicine and Molecular Imaging, Inc. the fifth with nephrolithiasis and hydronephrosis. Each patient received a standard adult dose of $370 \mathrm{MBq}(10 \mathrm{mCi})$ of ${ }^{99 \mathrm{~m}} \mathrm{Tc}-$ mercaptoacetyltriglycine intravenously with routine dynamic and sequential planar $\gamma$-camera imaging. Thirty minutes after radiotracer injection, $25 \mathrm{mg}$ of ethacrynic acid in $50 \mathrm{~mL}$ of $5 \%$ dextrose water were infused intravenously over $15 \mathrm{~min}$. Postdiuretic imaging was initiated after completion of the ethacrynic acid infusion and continued for $30 \mathrm{~min}$.

\section{RESULTS}

Of the 5 examinations, one was positive for a left renal collecting system obstruction (Fig. 1). The remaining 4 patients had no obstructive uropathy. Four patients had technically excellent diuretic renal images. Quantification of the half-time of radiotracer clearance was technically challenging in one patient because there was little activity in the

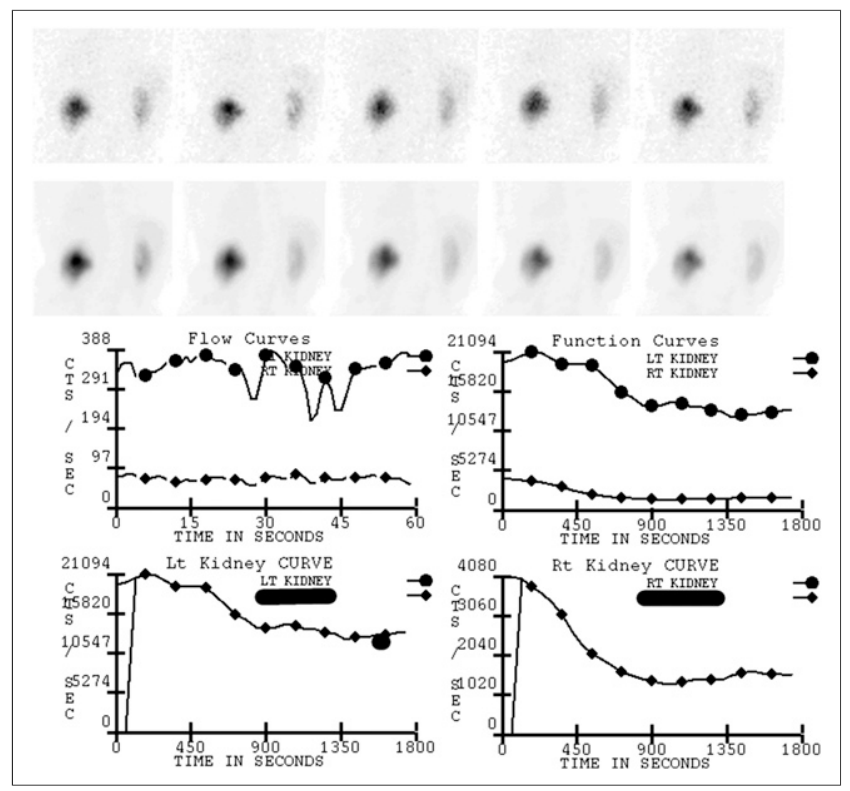

FIGURE 1. An 83-y-old man with severe sulfa allergy, benign prostatic hyperplasia, left hydronephrosis, and recurrent urinary tract infections. Prediuretic imaging (not shown) demonstrated prominent tracer activity in left pyelocaliceal structures suggestive of obstruction. Postdiuretic imaging with ethacrynic acid in upright position showed prolonged left renal half-time of 29 min with persistent hydronephrosis consistent with urodynamically significant obstruction. Findings for right kidney were normal. 


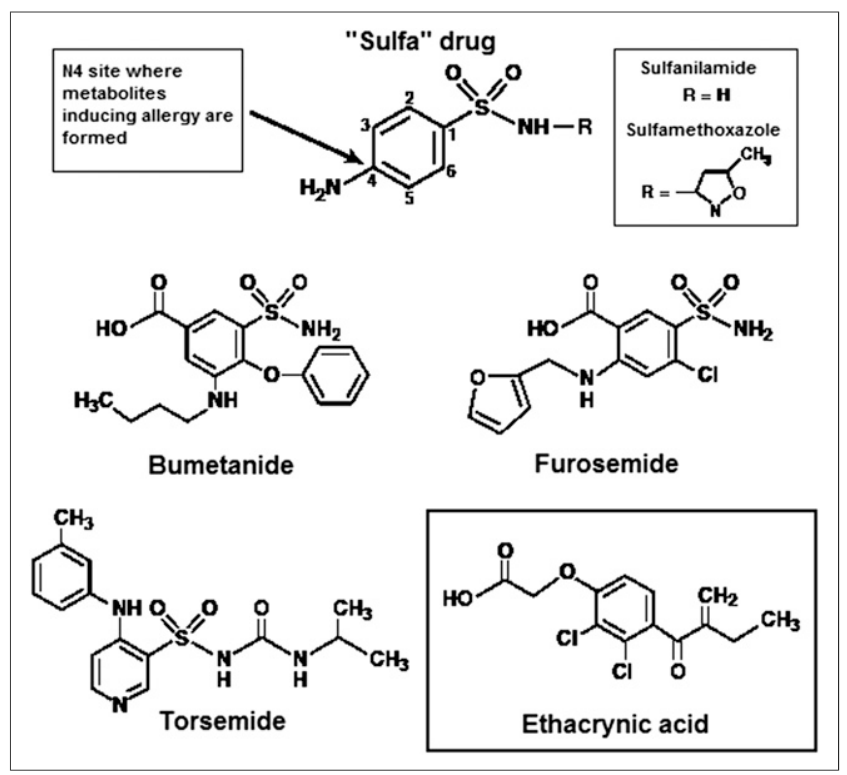

FIGURE 2. Sulfonamide drugs share common sulfur moiety $\left(\mathrm{SO}_{2} \mathrm{NHR}\right)$ attached to benzene ring. Sulfonamide antibiotics (upper boxes) have arylamine at N4 position, which incites metabolites responsible for allergic reactions. The diuretics bumetanide, furosemide, and torsemide have molecularly similar N4 sites that can cross-react and harm an allergic patient. Ethacrynic acid (lower box) does not contain a sulfonamide group.

collecting system yet no obstruction was visually apparent. There were no reported adverse reactions to ethacrynic acid.

\section{DISCUSSION}

In patients with a known severe allergy to sulfonamides, radiologists and nuclear medicine physicians face the difficult choice of whether to use furosemide for diuretic renal scintigraphy. Furosemide may induce sulfonamide hypersensitivity reactions ranging from fever to life-threatening events such as hepatic necrosis and Stevens-Johnson syndrome. Sulfonamide reactions occur in 3\%-6\% of the general population and in up to $60 \%$ of those with HIV (1). Although the mechanism may not be a true cross-reaction, patients with hypersensitivity reactions to antibiotic sulfonamides will react to nonantibiotic sulfonamides such as furosemide with a frequency approaching $10 \%(2-4)$. When the sulfonamideallergic patient presents for diuretic renal scintigraphy, there are 2 options: administering furosemide regardless of the known sulfamide reaction and relying on the rarity and often mildness of cross-reactions, or performing nondiuretic renal scintigraphy that cannot adequately evaluate for obstructive uropathy. A review for alternatives to furosemide revealed a sulfonamide moiety in many diuretics, including thiazides, carbonic anhydrase inhibitors, and most proximal loop diuretics, such as azosemide, bumetanide, and torsemide (5). The exception is ethacrynic acid, a rapidly acting loop diuretic available for intravenous administration (Fig. 2), making it an ideal alternative for renal scintigraphy of patients with severe sulfonamide allergies $(6,7)$. The major risk factor to consider when using intravenous ethacrynic acid is immediate volume depletion, a relevant life-threatening reaction for one-time use, requiring blood pressure monitoring. Attention to ototoxicity is important; temporary hearing loss and even deafness can result, particularly when ethacrynic acid is paired with aminoglycoside antibiotics. Less concerning reactions with repeated use include hypokalemic metabolic alkalosis and magnesium depletion $(8-10)$.

To confirm or rule out obstructive uropathy in patients with a known severe sulfonamide allergy, we developed a diagnostic imaging protocol for ethacrynic acid with input from our pharmacy department. Ethacrynic acid has nearly $100 \%$ bioavailability, with a steeper dose-response curve than furosemide. With intravenous injection, onset of action occurs in $5 \mathrm{~min}$, with the peak diuretic effect occurring within $30 \mathrm{~min}$ (11). Clinically, the weight-based dose of intravenous ethacrynic acid is $0.5-1 \mathrm{mg} / \mathrm{kg}$, not to exceed $100 \mathrm{mg}$ in a single dose (12). A typical patient with pulmonary edema may receive $25-50 \mathrm{mg}$ of ethacrynic acid intravenously over 20-30 min. To minimize the dose risk yet provide a brisk diuretic effect, we chose $25 \mathrm{mg}$ of ethacrynic acid in $50 \mathrm{~mL}$ of $5 \%$ dextrose water infused intravenously over a slightly shorter period of $15 \mathrm{~min}$. The postdiuretic image acquisitions were set to initiate after completion of ethacrynic acid infusion and to continue for $30 \mathrm{~min}$.

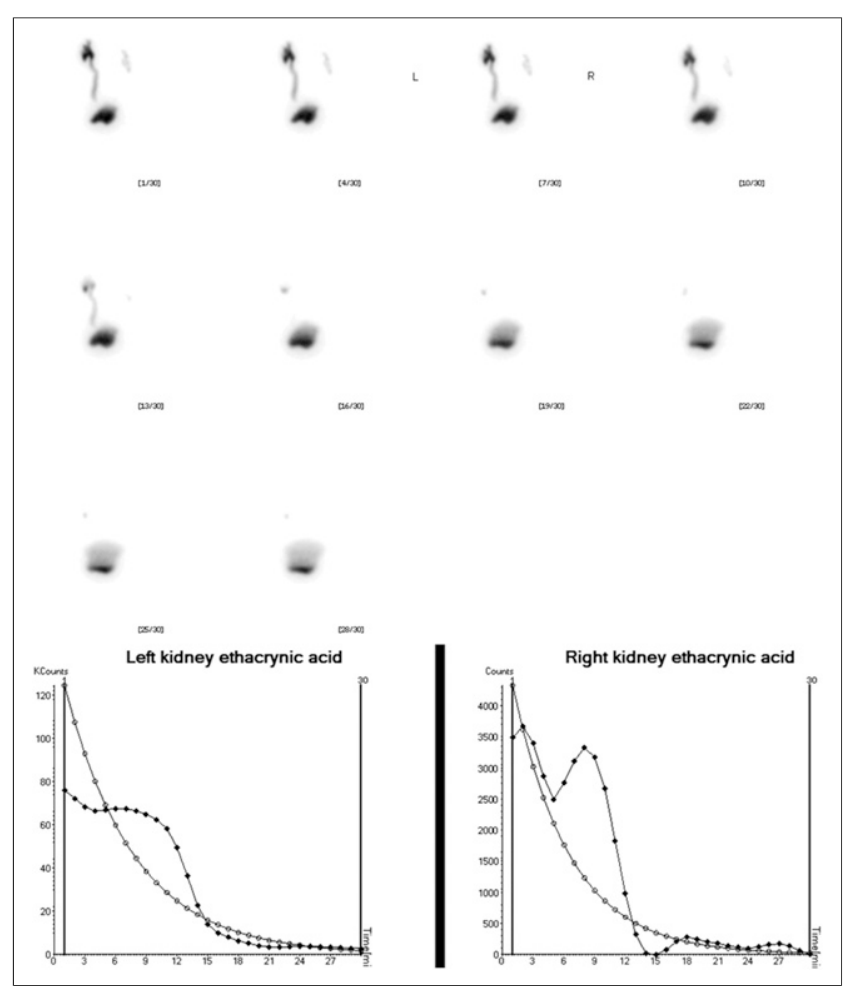

FIGURE 3. A 25-y-old man with severe sulfa allergy and history of urinary bladder extrophy-after closure, ureteral reimplantation, and subsequent appendicovesicostomy-presenting with left hydronephrosis and recurrent urinary tract infections. Prediuretic renal scintigraphy (not shown) demonstrated prominent activity in left collecting system suggestive of obstruction. Diuretic challenge using ethacrynic acid showed satisfactory emptying of both collecting systems, with half-times of 3.8 and $4.7 \mathrm{~min}$ on right and left sides, respectively, ruling out obstructive uropathy. 
Postdiuretic imaging of the 5 patients adequately answered the question of whether obstructive uropathy was present (Figs. 1 and 3). Our limited series further supports our initial short report on ethacrynic acid use for diuretic renal scintigraphy (13). The advantages of using ethacrynic acid in diuretic renal scintigraphy are its availability, relative simplicity of administration, absence of negative clinical impact for onetime use, and, most of all, ability to fully assess obstructive uropathy in patients with sulfonamide allergy. The main drawback resides in its cost of $\$ 300-\$ 600$ for a 25 -mg intravenous dose. Overall, when the diagnosis of obstructive uropathy is crucial for patient care, the cost of ethacrynic acid is probably counterbalanced by its imaging benefits, avoiding recourse to any other less efficient imaging modalities or invasive urologic procedures.

\section{CONCLUSION}

This study was successful as a proof of concept, and more research is needed to establish ethacrynic acid as a substitute for furosemide when patients with severe sulfonamide allergy must undergo diuretic scintigraphy.

\section{DISCLOSURE}

No potential conflict of interest relevant to this article was reported.

\section{REFERENCES}

1. Tilles SA. Practical issues in the management of hypersensitivity reactions: sulfonamides. South Med J. 2001;94:817-824.

2. Strom BL, Schinnar R, Apter AJ, et al. Absence of cross-reactivity between sulfonamide antibiotics and sulfonamide nonantibiotics. N Engl J Med. 2003; 349:1628-1635.

3. Wulf NR, Matuszewski KA. Sulfonamide cross-reactivity: is there evidence to support broad cross-allergenicity? Am J Health Syst Pharm. 2013;70: 1483-1494.

4. Brackett CC. Sulfonamide allergy and cross-reactivity. Curr Allergy Asthma Rep. 2007;7:41-48.

5. Reddy P, Mooradian AD. Diuretics: an update on the pharmacology and clinical uses. Am J Ther. 2009;16:74-85.

6. Wall GC, Bigner D, Craig S. Ethacrynic acid and the sulfa-sensitive patient. Arch Intern Med. 2003;163:116-117.

7. Ponka D. Approach to managing patients with sulfa allergy: use of antibiotic and nonantibiotic sulfonamides. Can Fam Physician. 2006;52:1434-1438.

8. O'Reilly PH; Consensus Committee of the Society of Radionuclides in Nephrourology. Standardization of the renogram technique for investigating the dilated upper urinary tract and assessing the results of surgery. BJU Int. 2003;91:239-243.

9. Hilson AJ. Functional renal imaging with nuclear medicine. Abdom Imaging. 2003;28:176-179.

10. Saremi F, Jadvar H, Siegel ME. Pharmacologic interventions in nuclear radiology: indications, imaging protocols, and clinical results. Radiographics. 2002;22: $477-490$.

11. Molnar J, Somberg JC. The clinical pharmacology of ethacrynic acid. Am J Ther 2009; 16:86-92.

12. Edecrin and sodium edecrin (ethacrynic acid and ethacrynate sodium) tablets and injection prescribing information [package insert]. Lawrenceville, NJ: Aton Pharma, Inc.; 2007.

13. Nguyen BD, Roarke MC, Howard OH. Ethacrynic acid as potential drug for diuretic renography in instance of sulfonamide allergy. Clin Nucl Med. 2010;35:547-549. 